\title{
Impact of uncertainties in the uranium 235 cross section resonance structure on characteristics measured in the BFS-79 critical assemblies
}

\author{
Olga Andrianova, Gleb Lomakov, and Gennady Manturov ${ }^{a}$ \\ JSC “SSC RF-IPPE”, Nuclear Reactors and Fuel Cycle Department, 249033 Bondarenko sq. 1, Obninsk, Russia
}

\begin{abstract}
The report presents the results of an analysis of benchmark experiments form the international ICSBEP Handbook (HEU-MET-INTER-005) carried out at the the SSC RF - IPPE in cooperation with the Idaho National Laboratory (INL, USA) applicable to the verification of calculations of a wide range of tasks related to safe storage of vitrified radioactive waste. Experiments on the BFS assemblies make it possible to perform a large series of studies needed for neutron data refinement, including measurements of reactivity effects which allow testing the neutron cross section resonance structure. This series of studies is considered as a sample joint analysis framework for differential and integral experiments required to correct nuclea data files of the ROSFOND evaluated neutron data library. Thus, it is shown that despite the wide range of available experimental data, in so far as it relates to the resonance region refinement, the experiments on reactivity measurement make it possible to more subtly reflect the resonance structure peculiarities in addition to the time-of-flight measurement method.
\end{abstract}

\section{Introduction}

Over the last few decades, data on BFS reactor physics experiments carried out at the SSC RF-IPPE have been widely used for correcting both nuclear data systems and software tools to be applied in calculation support for power and research reactors. Neutron cross-section data were obtained from the experiments carried out at different times with different BFS core configurations and compositions to examine the properties of fuel and structural materials [1]. These data were used for corrections of the ABBN-group constant system and the ROSFOND - Russian library of evaluated neutron data [2].

One of the current trends in reactor physics is to improve accuracy of reactor characteristics assessment by reducing the neutron data component in the calculation error. Sophisticated statistical approaches have been proposed and are nowadays widely used for the assessment of reactor characteristics uncertainties caused by nuclear data uncertainties [for instance, 3-5]. Especially valuable is that such approaches provide capabilities to propagate pointwise nuclear data uncertainty on reactor characteristics uncertainties that allows carrying out neutron crosssections adjustments with due account for both differential and integral experiments.

The article presents the results of a C/E discrepancy analysis of differential (measuring neutron transmission functions) and integral experiments (on the BFS critical assemblies) carried out to examine the properties of fuel and structural materials. Based on these results, proposals were made for correcting neutron data files of the ROSFOND library. The calculations were made using the MCNP transport code in point-wise neutron

a e-mail: mant@ippe.ru cross-section representation. As applied to calculations of neutron physics, the quality of calculations carried out using precision codes is determined by the reliability of neutron data files, because their application makes it possible to minimize the model uncertainty.

\section{Description of experimental program}

\subsection{Integral experiments on the BFS critical assemblies}

In cooperation with Idaho National Laboratory a program of critical experiments was executed on the BFS-1 facility at the SSC RF-IPPE. The program consisted of two series. The calculation models are described in the International Handbook of Evaluated Criticality Benchmark Safety Experiments $[6,7]$.

In the first series of experiments, the core was composed of aluminum pipes filled with ${ }^{235} \mathrm{U}$ pellets (90\%) and silicon dioxide. An analysis of a series of experiments on criticality perturbation measurements by introducing highly enriched ${ }^{235} \mathrm{U}$ samples of different sizes into the center of these assemblies showed that the results were significantly different from those obtained by means of precision calculated codes which use point-wise neutron cross sections.

The measurements in the BFS-79-5 assembles discovered that the reactivity introduced by ${ }^{235} \mathrm{U}(90 \%)$ pellets becomes negative. This effect was thoroughly examined [8]. It is obvious that this reactivity behavior may be due to the effect of ${ }^{235} \mathrm{U}$ resonance self-shielding within the intermediate neutron spectrum. First of all, the negative reactivity effect is caused by the core composition and configuration.

(c) The Authors, published by EDP Sciences. This is an Open Access article distributed under the terms of the Creative Commons Attribution License 4.0 (http://creativecommons.org/licenses/by/4.0/). 
In this paper, the experiments on the BFS, mainly composed of uranium and silicon, are used for correcting and verifying neutron data on uranium and silicon neutron cross-sections within the resonance region.

\subsection{Neutron transmission function measurements}

As part of the work, a large cycle of studies was considered in order to make a description of experiments on the IBR-2 fast pulsed reactor (Dubna, Russia) on measurements of neutron beam transmission through ${ }^{235} \mathrm{U}$ sample filters with the impurity content up to $10 \%$ (238 U-8.8\%, $234 \mathrm{U}-1.2 \%$ ). The results of these studies [9] were entered into the fundamental experiment database EXFOR (No.40082.2005) [10] and ICSBEP (FUND-JINR-1/EMULT-TRANS-001). The experiments were focused on the resonance self-shielding effects of ${ }^{235} \mathrm{U}$ sample filters during a neutron beam transmission; the energy range for total transmission measurements was within $0.1-200 \mathrm{keV}$.

Observed experimental data on ${ }^{235} \mathrm{U} \alpha$-value $(\alpha=$ $\left.\sigma_{\gamma} / \sigma_{\mathrm{f}}\right)$ has demonstrated the need for increasing the ${ }^{235} \mathrm{U}$ capture cross-section in the energy range from 500 to $2500 \mathrm{eV}$ in comparison with the recent evaluations. In addition to these experimental data, the results of benchmark experiments on uranium critical systems from the ICSBEP handbook also indicated on reasonableness to revise ${ }^{235} \mathrm{U}$ resonance parameters evaluation in the energy range $500-2500 \mathrm{eV}$.

\section{Methods and software tools used for nuclear data correction}

\subsection{Joint analysis of integral experiments for neutron data correction}

One of the current trends in reactor physics is improving the accuracy of predicted reactor performance by reducing the nuclear data uncertainty in the calculation error. Since new experiments require considerable time and resources, the most realistic way to reduce the nuclear data uncertainty in reactor characteristics would be to use evaluated neutron data obtained with due account for the total experimental information. It should be noted that this cycle of studies represents a suggested approach to neutron data corrections by sharing data of differential and integral experiments, and includes the following stages.

Firstly, sets of neutron data are formed based on the combinations of different evaluated data file sections, theoretical and statistical approaches; for further consideration only those sets are retained which have no significant disagreements with differential experiments.

Secondly, reactor characteristics and errors measured during the integral experiments on the critical systems are calculated using neutron transport codes according to the evaluated set of neutron data chosen with account of errors in the differential experiments.

Finally, the most appropriate (compromise) option of neutron data is chosen based on a complex comparison of $\mathrm{C} / \mathrm{E}$ discrepancies for the selected set of differential and integral experiments provided that it does not conflict with the proposed evaluation of neutron data.

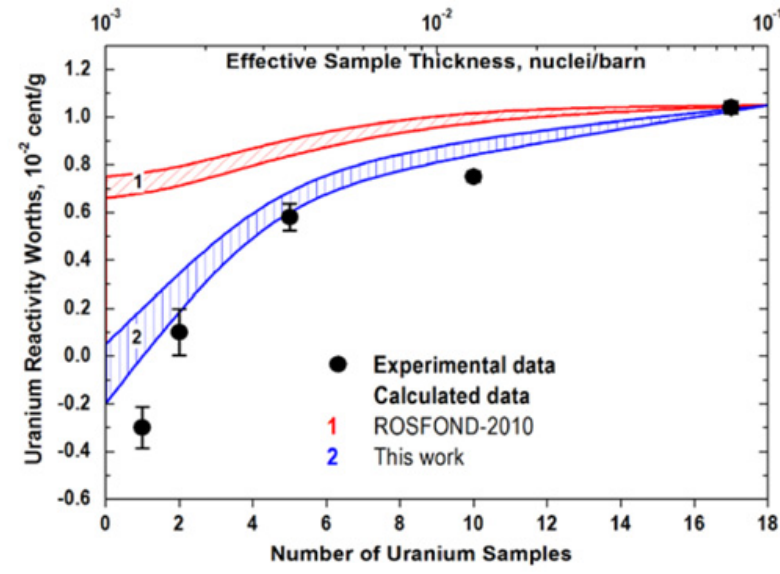

Figure 1. Central reactivity coefficients (shaded areas 1 and 2 indicate the calculation error associated with the uncertainty in resonance parameters).

Table 1. Calculation results for HMF-047 benchmark using original and modified (this work) ${ }^{235} \mathrm{U}$ ROSFOND data.

\begin{tabular}{|l|c|c|c|}
\hline Index & $\begin{array}{c}\text { Experiment } \\
\text { data [7] }\end{array}$ & $\begin{array}{c}\text { Nuclear } \\
\text { data }\end{array}$ & $\mathrm{C} / \mathrm{E}-1, \%$ \\
\hline$\sigma_{\mathrm{f}}\left({ }^{238} \mathrm{U}\right) /$ & 0.0150 & ROSFOND & $6.7 \pm 3.8$ \\
$\sigma_{\mathrm{f}}\left({ }^{235} \mathrm{U}\right)$ & \pm 0.005 & this work & $2.0 \pm 3.9$ \\
\hline$\sigma_{\mathrm{c}}\left({ }^{197} \mathrm{Au}\right) /$ & 1.15 & ROSFOND & $-21.7 \pm 4.9$ \\
$\sigma_{\mathrm{f}}\left({ }^{235} \mathrm{U}\right)$ & \pm 0.05 & this work & $-4.4 \pm 4.7$ \\
\hline
\end{tabular}

\subsection{Calculation models}

The calculation analysis was carried out using the MCNP transport code and the most up-to-date versions of nuclear data libraries (ROSFOND-2010, ENDF/B-VII.1, JEFF-3.2, and JENDL-4.0). Precision calculation models were made to the benchmark models for the experiments on neutron transmission through U samples in the ICSBEP. The models for the BFS assemblies from the ICSBEP were complemented with the calculations of reaction rates and central reactivity coefficients.

\section{Results and discussions}

\subsection{Resonance parameters correction for ${ }^{235} \mathrm{U}$}

Based on the methods of stochastic optimization and resonance statistics (Porter-Thomas and Wigner distributions), the ${ }^{235} \mathrm{U}$ resonance region (Leal et al., 2002 [11]) was revaluated within the energy range of $500-2000 \mathrm{eV}$. This made it possible to reduce the $\mathrm{C} / \mathrm{E}$ discrepancies for systems with the intermediate neutron spectrum and explain the detected effects (Fig. 1) associated with resonance neutron cross section self-shielding observed in the measurements on the BFS assemblies [12].

The options of corrected nuclear data were tested in the calculations of reactor characteristics measured on the BFS assemblies. A comparison of the results of the $\mathrm{C} / \mathrm{E}$ discrepancies in reactor characteristics for the suggested ${ }^{235} \mathrm{U}$ data is given in Table 1 and Fig. 2.

Figure 2 shows the calculated values of the effective multiplication factor obtained from different nuclear data libraries, depending on the configuration type, in increasing order of fissions within the thermal energy region (characteristic of the average neutron spectrum over the core). 


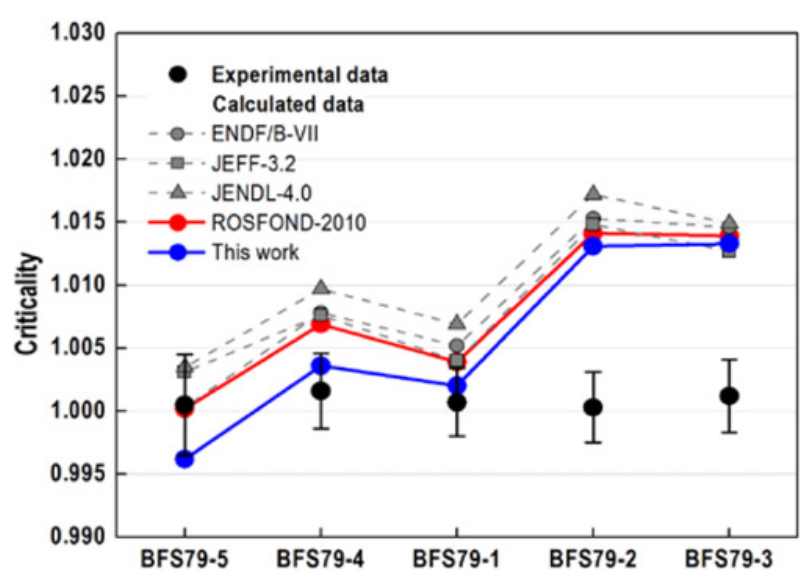

Figure 2. Calculated and experimental $k_{\text {eff }}$ for HMM-005 from different evaluated nuclear data libraries.

The calculation results show that the corrected data make it possible to significantly reduce the $\mathrm{C} / \mathrm{E}$ discrepancies even in the effective neutron multiplication factor for systems with the fast and intermediate neutron spectra.

In the case of systems with the thermal neutron spectrum, the corrections of neutron data do not reduce the $\mathrm{C} / \mathrm{E}$ discrepancies, as could be expected, since the modifications were introduced only into the resonance energy regions.

The authors are well aware that the proposed explanation of the detected discrepancies is probably not the only one possible. It is obvious that re-evaluation of the entire ${ }^{235} \mathrm{U}$ resonance region based on the new experimental data on differential and integral experiments may help to avoid the observed contradictions in the future descriptions of the detected experimental effects.

Nevertheless, it is the authors' opinion, that the observed discrepancies necessitate improving knowledge about the cross section resonance effects of the elements within the region of fast and intermediate neutron energies, where to find an acceptable evaluation will be a difficult task.

\section{Conclusions}

The discussed series of studies has exemplified an approach to evaluated nuclear data corrections based on the results of joint analysis of differential and integral experiments. As a part of the work, a new and more detailed evaluation of the BFS experiments was performed.
This makes it possible to perform a large series of studies needed for neutron data refinement. Inclusion measurements of reactivity effects allow testing the neutron cross section resonance structure.

The results of corrections of ${ }^{235} \mathrm{U}$ neutron resonance parameters obtained based on the stochastic optimization method and presented for the ROSFOND-2010 evaluated nuclear data library made it possible to give an explanation of the resonance cross section self-shielding effects observed in the experiments.

The authors express their gratitude to Prof. M. Nikolaev and V. Dulin, and Dr. Yu. Grigoriev for fruitful discussions during carrying out of this work.

\section{References}

[1] Andrianova O., Koscheev V., Lomakov G., Manturov G. PHYSOR 2016, Sun Valley, ID, May 1-5, 2166-2175 (2016)

[2] Zabrodskaya S.V., Ignatyuk A.V.,Koshcheev V.N., Manochin V.N., Nikolaev M.N., Pronyaev V.G. PAST. Ser.: NC, 1-2, 3 (2007) (available at http: // vant.ippe.ru/images/pdf/2007/1.pdf in russian)

[3] Hoefer A., Buss O., Hennebach M., Schmid M., Porsch D., Ann. Nucl. Energy 77, 514-521 (2015)

[4] Rochman, D., Koning, A., van der Mark, S., Hogenbirk, A., Sciolla, C., Ann. Nucl. Energy 38, 942-952 (2015)

[5] D. Rochman, W. Zwermann, S.C. van der Marck, A.J. Koning, H. Sjöstrand, P. Helgesson, B. Krzykacz-Hausmann, Nucl. Scien. \& Eng. 177(3), 337-349 (2014)

[6] Nikolaev M.; Tsiboulia A.; Matveenko I.; Briggs J. B.; Dean V. F., Trans. of the Am. Nucl. Soc. 84, 280 282 (2001)

[7] HEU-MET-MIXED-005, International Handbook of Evaluated Criticality Safety Benchmark Experiments, OECD NEA (2011)

[8] Pavlova O.N., Doulin V.A., Yadernaya energetika (2006) 2, 75-84 (in Russian)

[9] FUND-JINR-1/E-MULT-TRANS-001, International Handbook of Evaluated Criticality Safety Benchmark Experiments, OECD NEA (2011)

[10] EXFOR database - No.40082.2005

[11] Nikolaev M.N., Pavlova O.N. PAST. Ser.: NRC, is. 2-1, 70-79 (2006) (in Russian)

[12] Andrianova O.N. PhD dissertation (2015) (in russian) 